\title{
A Framework for Reasoning about Dynamic Axioms in Description Logics
}

\author{
Bartosz Bednarczyk $^{1,2}$, Stéphane Demri ${ }^{3}$, Alessio Mansutti ${ }^{3}$ \\ ${ }^{1}$ Computational Logic Group, TU Dresden \\ ${ }^{2}$ Institute of Computer Science, University of Wrocław \\ ${ }^{3}$ LSV, CNRS, ENS Paris-Saclay, Université Paris-Saclay
}

\begin{abstract}
Description logics are well-known logical formalisms for knowledge representation. We propose to enrich knowledge bases (KBs) with dynamic axioms that specify how the satisfaction of statements from the KBs evolves when the interpretation is decomposed or recomposed, providing a natural means to predict the evolution of interpretations. Our dynamic axioms borrow logical connectives from separation logics, well-known specification languages to verify programs with dynamic data structures. In the paper, we focus on $\mathcal{A L C}$ and $\mathcal{E} \mathcal{L}$ augmented with dynamic axioms, or to their subclass of positive dynamic axioms. The knowledge base consistency problem in the presence of dynamic axioms is investigated, leading to interesting complexity results, among which the problem for $\mathcal{E} \mathcal{L}$ with positive dynamic axioms is tractable, whereas $\mathcal{E} \mathcal{L}$ with dynamic axioms is undecidable.
\end{abstract}

\section{Introduction}

Nowadays, more and more digital information is stored but to make use of such data, it is common to employ logical formalisms to reason about knowledge and data. Description logics (DLs) [Baader et al., 2017] are among such formalisms that are intensively developed. In practical applications, the emerging data is changing rapidly. Hence, updating the knowledge bases (KBs) [Liu et al., 2006] in an efficient way is crucial for DLs in order to cope with real-life scenarios. Some effort was put to incorporate updates in DLs, e.g. in [Liu et al., 2006; De Giacomo et al., 2006; Drescher et al., 2009] or in [De Giacomo et al., 2017] for OBDA systems. Most of the cited works considered updates of the data layer (ABox) only, whereas we advocate that the logical rules used for reasoning may change too. Moreover, reasoning on updated ABoxes may require enriching the underlying DL language with new constructs, making unclear whether the existing DL reasoners can handle such new languages [De Giacomo et al., 2006]. Thus, there is still a need for novel DLs managing the updates on KBs.

In a broader perspective, the ability to specify in logical terms what are the effects of updating the models, is quite ubiquitous in computer science, with examples ranging from epistemic logics to specification languages for for- mal verification of programs. Prominent examples are logics of public announcements [Plaza, 1989] and separation logics [Reynolds, 2002]. The industrial success of separation logics, see e.g. [Distefano et al., 2019], is partly due to its ability to reason in a modular way on disjoint parts of the memory (by the means of the separating conjunction $*$ and the separating implication $*$ ). Roughly speaking, the operator $*$ decomposes the memory into two disjoint pieces and evaluates formulae on each piece separately. The operator $*$ asserts the behaviour of a heap after extending it with a new part satisfying certain properties. Though introduced in completely different contexts, some efforts on relating separation logics with DLs can be found in [Georgieva and Maier, 2005; Calvanese et al., 2014]. Many other logics concerning updates exist, e.g. sabotage and one-agent refinement modal logics [van Benthem, 2005; Bozzelli et al., 2013].

Motivations. Motivated by a lack of frameworks to specify evolution of interpretations in DLs, we would like to develop such formalisms by involving well-studied connectives from separation logics [Reynolds, 2002]. By contrast to the mentioned works on ABox updates, our framework aims at specifying how the satisfaction of GCIs or assertions evolves when the current interpretation is modified. Such an approach may benefit when ontologies are enriched by GCIs and assertions, either manually by the users or by automatic means. Herein, we plan to settle the foundations for such a framework.

Our contribution. We enrich KBs with dynamic axioms involving connectives from separation logics, that specify how the satisfaction of statements from the KBs evolves when the interpretation is decomposed or recomposed.

We introduce the notion of dynamic axioms (DAs) in KBs, endowing the set of interpretations with a binary composition operator $\oplus$, specifying that $\mathcal{J}=\mathcal{I}_{1} \oplus \mathcal{I}_{2}$ holds when $\mathcal{J}$ can be decomposed into $\mathcal{I}_{1}$ and $\mathcal{I}_{2}$. In this work, the operator $\oplus$ affects roles only, namely it decomposes each role into two disjoint pieces while keeping domains and the interpretation of concept names within the obtained interpretations unchanged. Our DAs feature dynamic connectives $*$ and - , respectively to decompose an interpretation and to augment it with a new disjoint interpretation. We distinguish the case of positive (i.e. negation-free) DAs. Throughout the paper, we study the KB consistency problem in the new setting.

For a logic $\mathfrak{L}$, we write $\mathfrak{L} \mathcal{D}\left(\right.$ resp. $\mathfrak{L D}^{+}$) to denote its ex- 
tension with DAs (resp. positive DAs). We show that consistency for $\mathcal{A L C D}{ }^{+}$KBs remains EXPTIME-complete by a translation into a known extension of $\mathcal{A L C}$. Moreover, the consistency for $\mathcal{A L C D} \mathrm{KBs}$ is proved undecidable by a reduction from the concept satisfiability of $\mathcal{A L C}$ extended by rolevalue maps. Surprisingly we show that undecidability transfers to $\mathcal{E} \mathcal{L} \mathcal{D}$. It is done by encoding concept negations within DAs. On the positive side, $\mathcal{E} \mathcal{L D}^{+}$is shown to be tractable, which makes it promising to implement.

\section{A Framework with Dynamic Axioms}

Basic DLs. We recall standard ingredients of the description logics (DLs) $\mathcal{A L C}$ and $\mathcal{E} \mathcal{L}$ [Baader et al., 2017], and then we present our new framework for reasoning about dynamic axioms. We fix countably infinite mutually disjoint sets of individual names $\mathbf{N}_{\mathbf{I}}$, concept names $\mathbf{N}_{\mathbf{C}}$, and role names $\mathbf{N}_{\mathbf{R}}$. The complex concepts of $\mathcal{A L C}$ are built from the grammar:

$$
C, C^{\prime}:=\top|A| \neg C\left|C \sqcap C^{\prime}\right| \exists r . C,
$$

where $A \in \mathbf{N}_{\mathbf{C}}$ is a concept name and $r \in \mathbf{N}_{\mathbf{R}}$ is a role name. The semantics of $\mathcal{A L C}$ concepts is defined via interpretations $\mathcal{I}=\left(\Delta^{\mathcal{I}},{ }^{\mathcal{I}}\right)$ composed of a non-empty set $\Delta^{\mathcal{I}}$ called the domain of $\mathcal{I}$ and a function ${ }^{\mathcal{I}}$ mapping individual names to elements of $\Delta^{\mathcal{I}}$, concept names to subsets of $\Delta^{\mathcal{I}}$, and role names to subsets of $\Delta^{\mathcal{I}} \times \Delta^{\mathcal{I}}$. This mapping is extended to complex concepts, according to the clauses below:

$$
\begin{gathered}
\top^{\mathcal{I}} \stackrel{\text { def }}{=} \Delta^{\mathcal{I}}, \quad(\neg C)^{\mathcal{I}} \stackrel{\text { def }}{=} \Delta^{\mathcal{I}} \backslash C^{\mathcal{I}}, \quad\left(C_{1} \sqcap C_{2}\right)^{\mathcal{I}} \stackrel{\text { def }}{=} C_{1}^{\mathcal{I}} \cap C_{2}^{\mathcal{I}}, \\
(\exists r . C)^{\mathcal{I}} \stackrel{\text { def }}{=}\left\{d \in \Delta^{\mathcal{I}} \mid \exists \text { e s.t. } e \in C^{\mathcal{I}},(d, e) \in r^{\mathcal{I}}\right\} .
\end{gathered}
$$

We use $\perp \stackrel{\text { def }}{=} \neg \top, \forall r . C \stackrel{\text { def }}{=} \neg \exists r . \neg C, C \sqcup C^{\prime} \stackrel{\text { def }}{=} \neg\left(\neg C \sqcap \neg C^{\prime}\right)$.

An assertion is an expression of the form $C(a), r(a, b)$ for $a, b \in \mathbf{N}_{\mathbf{I}}, C$ a complex concept and $r \in \mathbf{N}_{\mathbf{R}}$. A general concept inclusion (GCI) has the form $C_{1} \sqsubseteq C_{2}$. A statement is either a GCI, a concept assertion $C(a)$ or a role assertion $r(a, b)$. The satisfaction of a statement is recalled below.

$$
\mathcal{I} \models C_{1} \sqsubseteq C_{2} \stackrel{\text { def }}{\Leftrightarrow} C_{1}^{\mathcal{I}} \subseteq C_{2}^{\mathcal{I}},
$$

$\mathcal{I} \models C(a) \stackrel{\text { def }}{\Leftrightarrow} a^{\mathcal{I}} \in C^{\mathcal{I}}, \quad \mathcal{I} \models r(a, b) \stackrel{\text { def }}{\Leftrightarrow}\left(a^{\mathcal{I}}, b^{\mathcal{I}}\right) \in r^{\mathcal{I}}$. An $\mathcal{A L C}$ knowledge base $\mathcal{K}=(\mathcal{T}, \mathcal{A})$ consists of a finite set $\mathcal{A}$ (called $A B o x$ ) of assertions and a finite set $\mathcal{T}$ (called TBox) of GCIs. An interpretation $\mathcal{I}$ satisfies the $\mathrm{KB} \mathcal{K}=(\mathcal{T}, \mathcal{A})$ (or $\mathcal{I}$ is a model of $\mathcal{K}$, written: $\mathcal{I} \models \mathcal{K}$ ) if it satisfies all statements of $\mathcal{A}$ and $\mathcal{T}$. A knowledge base $\mathcal{K}$ is called consistent if it has a model. $\mathcal{E} \mathcal{L}$ is defined as the restriction of $\mathcal{A L C}$ to the concept constructor $\sqcap$, to the existential restriction $\exists$, to $\top$ and to the concept names. Definitions of $\mathcal{E} \mathcal{L}$ knowledge bases are lifted from $\mathcal{A} \mathcal{L C}$ knowledge bases in an obvious way.

Reasoning with DBoxes. We introduce axioms specifying properties of updated interpretations. We enrich the set of interpretations $\mathbb{I}$ with a composition operator $\oplus$, i.e. a partial function $\oplus: \mathbb{I} \times \mathbb{I} \rightarrow \mathbb{I}$ satisfying two natural properties:

(com) $\oplus$ is commutative, i.e. if $\mathcal{I} \oplus \mathcal{J}$ is defined, then $\mathcal{J} \oplus \mathcal{I}$ is defined and equal to $\mathcal{I} \oplus \mathcal{J}$.

(assoc) $\oplus$ is associative, i.e. if $\mathcal{I} \oplus(\mathcal{J} \oplus \mathcal{H})$ is defined, then $(\mathcal{I} \oplus \mathcal{J}) \oplus \mathcal{H}$ is defined and equal to $\mathcal{I} \oplus(\mathcal{J} \oplus \mathcal{H})$.

The function $\oplus$ is conceived as an operator to update dynamically the interpretations. For instance, when $\mathcal{J}=\mathcal{I}_{1} \oplus \mathcal{I}_{2}$ holds, we say that adding $\mathcal{I}_{2}$ to the interpretation $\mathcal{I}_{1}$ leads to the new interpretation $\mathcal{J}$, or alternatively, $\mathcal{J}$ can be decomposed into $\mathcal{I}_{1}$ and $\mathcal{I}_{2}$. In order to illustrate our approach, in this paper, only a specific composition operator is considered.

Whereas $\mathcal{A L C}$ KBs provide global constraints about the interpretations, we introduce dynamic boxes $\mathcal{D}$ (DBoxes) that specify how the satisfaction of statements evolve when the current interpretation is augmented or decomposed in the sense of the operator $\oplus$. For instance, $\mathcal{I}$ may not satisfy the GCI $(\exists r . \top \sqsubseteq \exists s . \top)$ but there is an additional interpretation $\mathcal{J}$ such that $\mathcal{I} \oplus \mathcal{J}$ is defined and satisfies $\exists r . \top \sqsubseteq \exists s . \top$. Typically, $\mathcal{J}$ contains new pairs of domain elements for the role $s$. Below, we define dynamic boxes as finite sets of $d y$ namic axioms and the above situation can be specified by

$$
\neg(\exists r . \top \sqsubseteq \exists s . \top) \sqcap(\top \circledast(\exists r . \top \sqsubseteq \exists s . \top)) .
$$

The operator $\circledast$ in $\mathbb{U}_{1} \circledast \mathbb{U}_{2}$ states that there is an interpretation satisfying $\mathbb{U}_{1}$ and whose composition with the current interpretation is defined so that the resulting (augmented) interpretation satisfies $\mathbb{U}_{2}$. Similarly, we consider the operator $*$ so that $\mathbb{U}_{1} * \mathbb{U}_{2}$ holds true when $\mathcal{I}$ can be decomposed into $\mathcal{J}_{1}$ and $\mathcal{J}_{2}$ (i.e. $\mathcal{I}=\mathcal{J}_{1} \oplus \mathcal{J}_{2}$ ), $\mathcal{J}_{1}$ satisfies $\mathbb{U}_{1}$ and $\mathcal{J}_{2}$ satisfies $\mathbb{U}_{2}$.

The set of positive dynamic axioms (PDAs) is defined by

$$
\begin{aligned}
\mathbb{U}, \mathbb{V}:= & \top|C(a)| r(a, b) \mid C \sqsubseteq D \\
& |\mathbb{U} \sqcup \mathbb{V}| \mathbb{U} \sqcap \mathbb{V}|\mathbb{U} * \mathbb{V}| \mathbb{U} \circledast \mathbb{V} .
\end{aligned}
$$

Consequently, positive dynamic axioms are built from GCIs, assertions and are closed under $\sqcap, \sqcup$ and under the dynamic connectives $*$ and $\circledast$ (no negation). If the underlying DL is restricted to a subset of concept constructors (as for instance $\mathcal{E} \mathcal{L}$ excludes $\sqcup$ ), the set of positive dynamic axioms is restricted accordingly. In full generality, dynamic axioms (DAs) are defined as Boolean combinations of positive dynamic axioms:

$$
U, V::=\mathbb{U}|\neg U| U \sqcup V \mid U \sqcap V .
$$

A (resp. positive) dynamic box (DBox) $\mathcal{D}$ is defined as a finite set of (resp. positive) dynamic axioms. Note that negation $\neg$ does not occur in the scope of $*$ and $\circledast$ and can therefore occur only on the top of positive dynamic axioms.

We define the satisfaction relation $\mid=$ between an interpretation and a dynamic axiom in the usual way for assertions, GCIs and for Boolean connectives. Here are the left clauses.

$$
\begin{aligned}
& \mathcal{I} \models \mathbb{U}_{1} * \mathbb{U}_{2} \quad \text { iff } \quad \text { there are } \mathcal{I}_{1}, \mathcal{I}_{2} \text { s.t. } \mathcal{I}=\mathcal{I}_{1} \oplus \mathcal{I}_{2} \text {, } \\
& \mathcal{I}_{1} \models \mathbb{U}_{1} \text { and } \mathcal{I}_{2} \models \mathbb{U}_{2} \\
& \mathcal{I} \models \mathbb{U}_{1} \circledast \mathbb{U}_{2} \quad \text { iff } \quad \text { there is } \mathcal{I}^{\prime} \text { s.t. } \mathcal{I} \oplus \mathcal{I}^{\prime} \text { is defined, } \\
& \mathcal{I}^{\prime}=\mathbb{U}_{1} \text { and } \mathcal{I} \oplus \mathcal{I}^{\prime}=\mathbb{U}_{2} \text {. }
\end{aligned}
$$

The dynamic connectives $*$ and $\circledast$ are exactly those used in separation logic [Reynolds, 2002; Vafeiadis and Parkinson, 2007] where $*$ is known as the separating conjunction and $\circledast$ is known as the septraction. A $K B$ with dynamic axioms is a triple $\mathcal{K}_{d a}=(\mathcal{T}, \mathcal{A}, \mathcal{D})$ where $(\mathcal{T}, \mathcal{A})$ forms a $\mathrm{KB}$ and $\mathcal{D}$ is a DBox. Similarly to the case of classical $\mathrm{KBs}$, we write $\mathcal{I} \models \mathcal{K}_{d a}$ (and say that $\mathcal{I}$ is a model of $\mathcal{K}_{d a}$ ) if for all the statements $\alpha$ from $(\mathcal{T} \cup \mathcal{A} \cup \mathcal{D})$, we have $\mathcal{I} \models \alpha$. A KB $\mathcal{K}_{d a}$ is said to be consistent if it has a model. Thus, the consistency problem is defined also for the dynamic setting.

Below, we introduce the interpretation composition that is used throughout the paper. We write $\mathcal{I}=\mathcal{I}_{1}+\mathcal{I}_{2}$ whenever three interpretations $\mathcal{I}, \mathcal{I}_{1}$ and $\mathcal{I}_{2}$ share the same domain, agree on the interpretation of the individual names and concept names, and for all $r \in \mathrm{N}_{\mathbf{R}}$, we have $r^{\mathcal{I}}=r^{\mathcal{I}_{1}} \uplus r^{\mathcal{I}_{2}}$, 
where $\uplus$ denotes disjoint union. Naturally, other operators could be investigated depending on the application domain.

Example 1. Let us consider a simple $K B \mathcal{K}_{d a}=(\mathcal{T}, \mathcal{A}, \mathcal{D})$ describing basketball teams and (possibly injured) basketball players. Let us assume that $\mathcal{T}$ contains the GCIs

\section{$\exists$ Gas_injury.Injury $\sqsubseteq$ Player, $\exists$ is_drafted.Team $\sqsubseteq P l a y e r$, $\exists$ has_injury.Injury $\sqcap \exists i$ is_drafted.Team $\sqsubseteq \perp$,} essentially stating that no injured player can be drafted by a team. For the data layer, we suppose that the ABox contains has_injury("Zion", "knee"). We can infer in a natural way that there is an evolution where the player "Zion" is drafted by the team "Pelicans" by asking for the consistency of $\mathcal{K}_{d a}$ in the case where $\mathcal{D}$ contains the dynamic axiom

$\top *\left(\top-\right.$ is_drafted( “Zion”, "Pelicans”) $\left.\sqcap \prod_{\alpha \in \mathcal{T}} \alpha\right)$. Notably, an interpretation $\mathcal{I}$ satisfying $\mathcal{K}_{d a}$ can be decomposed into $\mathcal{I}^{\prime}+\mathcal{J}$ where $\mathcal{I}^{\prime}=$ has_injury( "Zion", "knee"). Then, $\mathcal{J}$ (the interpretation where "Zion" is not injured) is augmented so that is_drafted("Zion", "Pelicans") holds, leading to the satisfaction of the dynamic axiom above. The PDA $\prod_{\alpha \in \mathcal{T}} \alpha$ is added to guarantee that $\mathcal{J}$ satisfies the TBox.

Given a description logic $\mathfrak{L}$ (in particular specifying a set of concepts and a set of roles), we write $\mathfrak{L D}^{+}$to denote its extension with positive dynamic axioms based on the concepts and roles from $\mathfrak{L}$, the concept constructors from $\mathfrak{L}$ and based on $(\mathbb{I},+)$. Similarly, we write $\mathfrak{L} \mathcal{D}$ to denote its extension with dynamic axioms and based on $(\mathbb{I},+)$. We write $\operatorname{CONS}(\mathfrak{L})$ to denote the knowledge base consistency problem for $\mathfrak{L}$. In the rest of the paper, we investigate the decidability status or the computational complexity of the knowledge base consistency problem for the logics $\mathcal{E} \mathcal{L} \mathcal{D}^{+}, \mathcal{E} \mathcal{L} \mathcal{D}, \mathcal{A L C D}{ }^{+}$and $\mathcal{A L C D}$.

\section{A Toolkit for Positive Dynamic Axioms}

In this section, we provide developments about the consistency of positive dynamic axioms $\mathbb{U}$ that are helpful to characterise the complexity of $\operatorname{CONS}\left(\mathcal{A L C D}{ }^{+}\right)$and $\operatorname{CONS}\left(\mathcal{E} \mathcal{L D}^{+}\right)$. Below, $\mathfrak{L}$ is either $\mathcal{A L C D} \mathcal{D}^{+}$or $\mathcal{E} \mathcal{L} \mathcal{D}^{+}$. The culminating result is forthcoming Lemma 1 that states how the consistency of $\mathbb{U}$ can be expressed as the existence of a family of interpretations satisfying disjointness constraints.

Axioms as finite trees. Positive dynamic axioms can be represented as labelled finite binary trees using the standard tree encoding for terms. We recall that a binary tree Tree, which may contain nodes with (only) one child, is a nonempty subset of $\{1,2\}^{*}$ such that, for all $\mathfrak{n} \in\{1,2\}^{*}$ and $i \in$ $\{1,2\}, \mathfrak{n} \cdot i \in$ Tree implies $\mathfrak{n} \in$ Tree and, $\mathfrak{n} \cdot 2 \in$ Tree implies $\mathfrak{n} \cdot 1 \in$ Tree. The elements of Tree are called nodes. The root of Tree is $\varepsilon$, i.e. the empty word. A (positive) dynamic axiom $\mathbb{U}$ can be represented by a labelled finite binary tree $\mathfrak{f}_{\mathbb{U}}: \operatorname{Tree}_{\mathbb{U}} \rightarrow \operatorname{sub}(\mathbb{U})$, where

- $\operatorname{sub}(\mathbb{U})$ is the set of subaxioms of $\mathbb{U}$, defined as usually. For instance, for $\mathbb{U}=r(a, b) \sqcap(r(a, b) \circledast D(b))$, we have $\operatorname{sub}(\mathbb{U})=\{r(a, b), r(a, b) \circledast D(b), D(b), \mathbb{U}\}$.

- Tree $\mathbb{U}_{\mathbb{U}}$ is the tree satisfying the following constraints:

- $\mathfrak{f}_{\mathbb{U}}(\varepsilon)=\mathbb{U}$; if $\mathfrak{f}_{\mathbb{U}}(\mathfrak{n})$ is atomic, then $\mathfrak{n}$ is a leaf,

- if $\mathfrak{f}_{\mathbb{U}}(\mathfrak{n})=\mathbb{U}_{1} \otimes \mathbb{U}_{2}$ for some binary $\otimes$, then $\mathfrak{n} \cdot 1$ and $\mathfrak{n} \cdot 2$ are in Tree $\mathbb{U}$ with $\mathfrak{f}_{\mathbb{U}}(\mathfrak{n} \cdot 1)=\mathbb{U}_{1}$ and $\mathfrak{f}_{\mathbb{U}}(\mathfrak{n} \cdot 2)=\mathbb{U}_{2}$.
Nodes associated with interpretations. Some nodes of Tree $_{\mathbb{U}}$ will be associated with interpretations and constraints between those interpretations should be satisfied (see also the definition of Disj $\mathbb{U}$ below as well as Lemma 1). We write Int $_{\mathbb{U}}$ to denote the smallest subset of Tree $\mathbb{U}_{\mathbb{U}}$ such that $\varepsilon \in \operatorname{Int}_{\mathbb{U}}$ and $\mathfrak{n} \cdot 1, \mathfrak{n} \cdot 2 \in \operatorname{Int}_{\mathbb{U}}$, for $\mathfrak{n}$ having $\mathfrak{f}_{\mathbb{U}}(\mathfrak{n})$ of the form either $\mathbb{U}_{1} \circledast \mathbb{U}_{2}$ or $\mathbb{U}_{1} * \mathbb{U}_{2}$. For example, with $\mathbb{U}^{\star}=$ $\left(\mathbb{U}_{1} \circledast \mathbb{U}_{2}\right) \sqcap\left(\mathbb{U}_{1}^{\prime} \circledast \mathbb{U}_{2}^{\prime}\right)$, we have Int $\mathbb{U}^{\star} \supseteq\{\varepsilon, 1 \cdot 1,1 \cdot 2,2 \cdot 1,2 \cdot 2\}$. Informally speaking, the nodes in Int $_{\mathbb{U}}$ correspond to subaxioms of $\mathbb{U}$ that require the introduction of different interpretations. Thus, elements of Int $\mathbb{U}$ are implicitly associated with interpretations that relate them, as formally described below with the definition of $\operatorname{Disj}_{\mathbb{U}}$. Given $\mathfrak{n} \in \operatorname{Tree}_{\mathbb{U}}$, we write $\mathfrak{i}(\mathfrak{n})$ to denote the maximal prefix of $\mathfrak{n}$ that is in $\operatorname{lnt}_{\mathbb{U}}$. With $\mathbb{U}^{\star}$ above, we have $\mathfrak{i}(1)=\varepsilon$ and $\mathfrak{i}(2)=\varepsilon$ but $\mathfrak{i}(1 \cdot 1)=1 \cdot 1$.

Disjointness axioms. We define the set of disjointness axioms Disj $j_{\mathbb{U}}$ using the nodes from $\operatorname{lnt} t_{\mathbb{U}}$. As stated above, each element of $\operatorname{lnt}_{\mathbb{U}}$ is associated with an interpretation. Because of this, these elements must satisfy expressions of the form $\mathfrak{n}=\mathfrak{n}_{1}+\mathfrak{n}_{2}$, that reflect constraints between the corresponding interpretations. We overload the symbol ' + ' but we hope that this does not lead to any confusion. The set Disj $\mathbb{U}$ is the smallest set of disjointness axioms of the form $\mathfrak{n}=\mathfrak{n}_{1}+\mathfrak{n}_{2}$ with $\mathfrak{n}, \mathfrak{n}_{1}, \mathfrak{n}_{2} \in \operatorname{Int}_{\mathbb{U}}$ such that

- if $\mathfrak{f}_{\mathbb{U}}(\mathfrak{n})=\mathbb{U}_{1} * \mathbb{U}_{2}$ then $\mathfrak{i}(\mathfrak{n})=(\mathfrak{n} \cdot 1)+(\mathfrak{n} \cdot 2) \in \operatorname{Disj}_{\mathbb{U}}$,

- if $\mathfrak{f}_{\mathbb{U}}(\mathfrak{n})=\mathbb{U}_{1} \circledast \mathbb{U}_{2}$ then $(\mathfrak{n} \cdot 2)=(\mathfrak{n} \cdot 1)+\mathfrak{i}(\mathfrak{n}) \in$ Disj $_{\mathbb{U}}$,

- if $\mathfrak{n}=\mathfrak{n}_{1}+\mathfrak{n}_{2} \in \operatorname{Disj}_{\mathbb{U}}$ then $\mathfrak{n}=\mathfrak{n}_{2}+\mathfrak{n}_{1} \in \operatorname{Disj}_{\mathbb{U}}$.

As the dynamic connectives $*$ and $\circledast$ have an existential flavour, satisfaction of $\mathbb{U}$ on an interpretation implies the existence of several interpretations constrained by compositions. The set of constraints between all these interpretations is represented by the set Disj $j_{\mathbb{U}}$ of disjointness axioms of the form $\mathfrak{n}=\mathfrak{n}_{1}+\mathfrak{n}_{2}$. For instance, with $\mathbb{U}^{\star}$ above, we have

$$
\operatorname{Disj}_{\mathbb{U} \star} \supseteq\{1 \cdot 2=\varepsilon+1 \cdot 1,2 \cdot 2=\varepsilon+2 \cdot 1,1 \cdot 2=1 \cdot 1+\varepsilon\} .
$$

More precisely, the sets $\operatorname{Disj}_{\mathbb{U}}$ and $\operatorname{lnt}_{\mathbb{U}}$ are related as follows. A map $\mathfrak{g}: \operatorname{lnt} \mathbb{U} \rightarrow \mathbb{I}$ is said to be a complete witness for $\mathbb{U}$ iff for all $\mathfrak{n} \in \operatorname{lnt} \mathbb{U}$, we have $\mathfrak{g}(\mathfrak{n}) \models \mathfrak{f}_{\mathbb{U}}(\mathfrak{n})$ and if $\mathfrak{n}=\mathfrak{n}_{1}+\mathfrak{n}_{2}$ is in $\operatorname{Disj}_{\mathbb{U}}$, then $\mathfrak{g}(\mathfrak{n})=\mathfrak{g}\left(\mathfrak{n}_{1}\right)+\mathfrak{g}\left(\mathfrak{n}_{2}\right)$. When $\mathfrak{g}$ is a complete witness, $\mathfrak{g}(\varepsilon) \models \mathbb{U}$. Not only $\mathfrak{g}(\varepsilon)$ is a model for $\mathbb{U}$ but $\mathfrak{g}$ determines completely how interpretations are decomposed or recomposed to guarantee the satisfaction of $\mathfrak{g}(\varepsilon) \models \mathbb{U}$.

Lemma 1. Let $\mathbb{U}$ be a positive dynamic axiom in $\mathfrak{L}$. $\mathbb{U}$ is consistent iff there is a complete witness for $\mathbb{U}$.

Note that the lemma can be applied to $\mathcal{E} \mathcal{L D}^{+}$and $\mathcal{A L C D ^ { + }}$. The "only if" part is obvious, since $\mathfrak{g}(\varepsilon)=\mathbb{U}$. For the "if" direction, the proof is by induction on the number of occurrences of either $*$ or $\circledast$ in $\mathbb{U}$. Actually, we can show that if $\mathcal{I}=\mathbb{U}$, then there is a complete witness for $\mathbb{U}$ with $\mathfrak{g}(\varepsilon)=\mathcal{I}$. When $\mathbb{U}$ is $* / \circledast$-free, Int $_{\mathbb{U}}=\{\varepsilon\}$ and Disj $j_{\mathbb{U}}$ is empty, which obviously leads to the conclusion. Otherwise, we perform a standard case analysis, the details are omitted.

\section{Reasoning on $\mathcal{A L C}$ with Dynamic Axioms}

We study the effect of adding dynamic boxes to $\mathcal{A L C}$, resulting in description logics $\mathcal{A L C D}{ }^{+}$and $\mathcal{A L C D}$. 


\subsection{Positive Dynamic Axioms for $\mathcal{A L C}$}

Below, we show that the consistency problem for $\mathcal{A L C D}^{+}$ is EXPTIME-complete, knowing that EXPTIME-hardness is inherited from $\mathcal{A L C}$. The upper bound EXPTIME is obtained by internalising $\operatorname{CONS}\left(\mathcal{A L C D}{ }^{+}\right)$within the description logic $\mathcal{A L C O} b_{\text {reg }}^{\text {self }}($ a.k.a. $\mathcal{Z O}$ ) for which knowledge base consistency is known to be in EXPTIME [Calvanese et al., 2009, Theorem 3.11]. The DL $\mathcal{Z O}$ extends $\mathcal{A L C}$ in many directions, but for our purposes, we recall the features that are essential for our reduction. $\mathcal{Z O}$ contains the universal role $\mathrm{T}$ (i.e. $\mathrm{T}^{\mathcal{I}}=\Delta^{\mathcal{I}} \times \Delta^{\mathcal{I}}$ ), nominals $\{o\}$ (i.e. $\{o\}^{\mathcal{I}}$ is a singleton), and complex roles of the form $r,\left(\left(r_{1} \cup r_{2}\right) \backslash r\right),\left(r \backslash\left(r_{1} \cup r_{2}\right)\right)$ and $\left(r_{1} \cap r_{2}\right)$. For instance, expressing $r^{\mathcal{I}}=r_{1}^{\mathcal{I}} \uplus r_{2}^{\mathcal{I}}$ can be done with the following set of GCIs $\left[r=r_{1} \uplus r_{2}\right]$ in $\mathcal{Z O}$ :

$\left\{\top \sqsubseteq \forall\left(r_{1} \cap r_{2}\right) \cdot \perp, \top \sqsubseteq \forall\left(\left(r_{1} \cup r_{2}\right) \backslash r\right) \cdot \perp, \top \sqsubseteq \forall\left(r \backslash\left(r_{1} \cup r_{2}\right)\right) . \perp\right\}$

Let $\mathcal{K}_{d a}=(\mathcal{T}, \mathcal{A}, \mathcal{D})$ be an $\mathcal{A L C D}{ }^{+}$knowledge base with positive dynamic axioms. We write $\mathbb{U}^{\star}$ to denote the positive dynamic axiom $\prod_{\alpha \in \mathcal{T} \cup \mathcal{A} \cup \mathcal{D}} \alpha$ and $R$ to denote the set of role names occurring in $\mathbb{U}^{\star}$. Notice that $\mathcal{K}_{d a}$ and the $\mathcal{A} \mathcal{L C} \mathcal{D}^{+}$ knowledge base $\left(\emptyset, \emptyset,\left\{\mathbb{U}^{\star}\right\}\right)$ are equiconsistent. It is time to take advantage of Section 3, and rely on the notions of $\operatorname{lnt}_{\mathbb{U}^{*}}$ and Disj $j_{\mathbb{}}$ therein introduced. The correctness of the construction is then guaranteed by Lemma 1 . Given $\mathfrak{n} \in \ln \mathbb{U}_{\mathbb{U}^{\star}}$, we write $C^{\mathfrak{n}}$ to denote the concept obtained from $C$ but in which each role name $r$ is substituted by a new role name $r^{\mathfrak{n}}$ (nothing is done on the concept names). Intuitively, given a complete witness $\mathfrak{g}$ for $\mathbb{U}^{\star}$, the $\mathcal{Z O}$ interpretation of $r^{\mathfrak{n}}$ corresponds to the interpretation of $r$ in $\mathfrak{g}(\mathfrak{n})$, consistently with the translation $\tau$ defined below. Assertions and GCIs are translated as usually in the presence of the universal role and nominals, whereas, as a peculiarity, the constraints about disjointness are stated in the target $\mathcal{Z O}$ TBox (see the last clause).

$$
\begin{aligned}
& \text { - } \tau(C(a), \mathfrak{n}) \stackrel{\text { def }}{=} \forall \mathrm{T} .\left(\neg\left\{o_{a}\right\} \sqcup C^{\mathrm{i}(\mathfrak{n})}\right), \\
& \text { - } \tau(r(a, b), \mathfrak{n}) \stackrel{\text { def }}{=} \forall \mathrm{T} .\left(\neg\left\{o_{a}\right\} \sqcup \exists r^{\mathrm{i}(\mathfrak{n})} \cdot\left\{o_{b}\right\}\right), \\
& \text { - } \tau(C \sqsubseteq D, \mathfrak{n}) \stackrel{\text { def }}{=} \forall \mathrm{T} .\left(\neg C^{\mathfrak{i}(\mathfrak{n})} \sqcup D^{\mathfrak{i}(\mathfrak{n})}\right), \\
& \text { - } \tau(\mathbb{U} \otimes \mathbb{V}, \mathfrak{n}) \stackrel{\text { def }}{=} \tau(\mathbb{U}, \mathfrak{n} \cdot 1) \otimes \tau(\mathbb{V}, \mathfrak{n} \cdot 2) \text { with } \otimes \in\{\sqcup, \sqcap\} . \\
& \text { - } \tau(\mathbb{U} \otimes \mathbb{V}, \mathfrak{n}) \stackrel{\text { def }}{=} \tau(\mathbb{U}, \mathfrak{n} \cdot 1) \sqcap \tau(\mathbb{V}, \mathfrak{n} \cdot 2) \text { with } \otimes \in\{*,-\circledast\} .
\end{aligned}
$$

In the first three cases above, we rely on the fact that for every $\mathcal{I}=\left(\Delta^{\mathcal{I}},{ }^{\mathcal{I}}\right)$, if $C^{\mathcal{I}} \subseteq D^{\mathcal{I}}$ then $(\forall \mathrm{T} . \neg C \sqcup D)^{\mathcal{I}}=\Delta^{\mathcal{I}}$, else $(\forall \mathrm{T} . \neg C \sqcup D)^{\mathcal{I}}=\emptyset$. The correctness of $\tau$ is stated below.

Lemma 2. $\mathcal{K}_{d a}$ is consistent iff $(\mathcal{T}, \mathcal{A})$ is consistent with $\mathcal{T}=$ $\bigcup\left\{\left[r^{\mathfrak{n}}=r^{\mathfrak{n}_{1}} \uplus r^{\mathfrak{n}_{2}}\right] \mid \mathfrak{n}=\mathfrak{n}_{1}+\mathfrak{n}_{2} \in \operatorname{Disj}_{\mathbb{U}^{\star}}, r \in R\right\}$ and $\mathcal{A}=$ $\left\{\tau\left(\mathbb{U}^{\star}, \varepsilon\right)(a)\right\}$, where $a$ is an arbitrary individual name.

In the proof, an interpretation satisfying $(\mathcal{T}, \mathcal{A})$ can be read as a complete witness for $\mathbb{U}$ and by Lemma 1 this entails the consistency of $\mathcal{K}_{d a}$. The converse direction is proved analogously. As the knowledge base in $\mathcal{Z O}$ is of polynomial-size in the size of $\mathcal{K}_{d a}$, we get the EXPTIME upper bound.

Theorem 1. $\operatorname{CONS}\left(\mathcal{A L C D}{ }^{+}\right)$is EXPTIME-complete.

\subsection{Undecidability of $\operatorname{CONS}(\mathcal{A L C D})$}

We have seen that $\operatorname{CONS}\left(\mathcal{A L C D}{ }^{+}\right)$is EXPTIME-complete. By contrast, we show that adding negation at the top-level of PDAs leads to the undecidability of $\operatorname{CONS}(\mathcal{A L C D})$.
To start with, we consider the well-known extension of $\mathcal{A L C}$ with complex role inclusion axioms (CRIAs) of the form $r_{1} \circ \cdots \circ r_{n} \sqsubseteq s$, where $\mathcal{I} \models r_{1} \circ \cdots \circ r_{n} \sqsubseteq s$ iff $r_{1}^{\mathcal{I}} \circ \cdots \circ r_{n}^{\mathcal{I}} \subseteq s^{\mathcal{I}}$. An $R B o x \mathcal{R}$ is a finite collection of CRIAs. A concept $C$ in $\mathcal{A L C}$ is satisfiable with respect to the RBox $\mathcal{R}$ iff there is $\mathcal{I}$ such that $C^{\mathcal{I}} \neq \emptyset$ and for all $\alpha \in \mathcal{R}$, we have $\mathcal{I} \models \alpha$. Without regularity conditions on the RBox $\mathcal{R}$, as considered in [Demri, 2001; Horrocks and Sattler, 2004], satisfiability of $\mathcal{A L C}$ concepts with respect to RBoxes made of arbitrary CRIAs is undecidable, see e.g. [Baldoni et al., 1998]. This is precisely the problem reduced to $\operatorname{CONS}(\mathcal{A L C D})$.

In order to encode a CRIA of the form $r_{1} \circ \cdots \circ r_{n} \sqsubseteq s$ into a dynamic axiom, we take advantage of auxiliary and fresh role names $t, t_{1}, t_{2}$ interpreted by the empty relation. Note that GCIs are atomic positive dynamic axioms and the GCI $\exists t . \top \sqsubseteq \perp$ holds only in interpretations $\mathcal{I}$ such that $t^{\mathcal{I}}=\emptyset$. To better reflect this, we use $t \equiv \emptyset$ to denote $\exists t . \top \sqsubseteq \perp$.

Let $C$ be a concept in $\mathcal{A L C}$ written with role names occurring in $\left\{s_{1}, \ldots, s_{m}\right\}$. As a first step for the translation, we introduce a negation-free dynamic axiom that is roughly equivalent to $\neg(C \sqsubseteq \perp)$, i.e. it is satisfied only by interpretations $\mathcal{I}$ such that $C^{\overline{\mathcal{I}}} \neq \emptyset$. As it is negation-free, this dynamic axiom can be then used under the scope of $\circledast$ and $*$. In order to define such a dynamic axiom, we rely on an auxiliary role name $t$ (not occurring in $C$ ). Then, we write $\left\langle C \not_{t} \perp\right\rangle$ to denote the (positive) dynamic axiom below:

$$
\left(\prod_{r \in\left\{s_{1}, \ldots, s_{m}\right\}}(r \equiv \emptyset)\right)-(\top \sqsubseteq \exists t . C)
$$

Informally, under the assumption that $t$ is interpreted vacuously, this formula ensures that at least one domain element satisfies $C$ (because the disjoint new interpretation added through $\circledast$ can be defined so that every domain element points to some element satisfying $C$, via $t$ ).

Lemma 3. Let $\mathcal{I}$ be an interpretation such that $t^{\mathcal{I}}=\emptyset$ holds. Then $C^{\mathcal{I}}$ is non-empty iff $\mathcal{I} \models\left\langle C \not \equiv_{t} \perp\right\rangle$ holds.

Let us explain now how to encode a CRIA $\vec{r} \sqsubseteq s$, where $\vec{r}=r_{1} \circ \cdots \circ r_{n}$, starting with its negation. We write $V\left(\vec{r}, s, t_{1}, t_{2}\right)$ to denote the dynamic axiom below:

$\left(\prod(r \equiv \emptyset)\right)-\circledast\left(t_{2} \equiv \emptyset \sqcap\left\langle\exists r_{1} \cdots \exists r_{n} \exists t_{1} \cdot \top \sqcap \neg \exists s \cdot \exists t_{1} \cdot \top \not \equiv_{t_{2}} \perp\right\rangle\right)$ $r \in\left\{r_{1}, \ldots, r_{n}, s\right\}$

Let us consider an interpretation $\mathcal{I}$ such that $t_{1}^{\mathcal{I}}=t_{2}^{\mathcal{I}}=\emptyset$. Informally, this dynamic axiom simply states that when $(d, e) \in$ $\left(r_{1} \circ \cdots \circ r_{n}\right)^{\mathcal{I}}$ but $(d, e) \notin s^{\mathcal{I}}$ for some $d, e$, it is possible to augment $t_{1}^{\mathcal{I}}$ with the interpretation $\mathcal{J}$ such that $(d, f) \in$ $\left(r_{1} \circ \cdots \circ r_{n} \circ t_{1}\right)^{\mathcal{I}+\mathcal{J}}$ for some $f$ but $\left(d, f^{\prime}\right) \notin\left(s \circ t_{1}\right)^{\mathcal{I}+\mathcal{J}}$ for all $f^{\prime}$. Also, observe that $V\left(\vec{r}, s, t_{1}, t_{2}\right)$ uses negation only in $\mathcal{A L C}$ subconcepts (see $\neg \exists s . \exists t_{1} \cdot \top$ ). The negation of $V\left(\vec{r}, s, t_{1}, t_{2}\right)$ captures the concept of CRIAs as follows.

Lemma 4. Let $\mathcal{I}$ be s.t. $t_{1}^{\mathcal{I}}=t_{2}^{\mathcal{I}}=\emptyset$. Let $\vec{r} \sqsubseteq s$ be a CRIA, where $\vec{r}=r_{1} \circ \cdots \circ r_{n} . \mathcal{I}=\neg V\left(\vec{r}, s, t_{1}, t_{2}\right) \overline{\text { iff }} \mathcal{I}=\vec{r} \sqsubseteq s$.

Consequently, given an $\mathcal{A L C}$ concept $C$, an RBox $\mathcal{R}$ and an individual name $a, C$ is satisfiable with respect to $\mathcal{R}$ iff the following dynamic axiom in $\mathcal{A L C D}$ is consistent:

$$
C(a) \sqcap\left(t_{1} \equiv \emptyset\right) \sqcap\left(t_{2} \equiv \emptyset\right) \sqcap \prod_{\vec{r} \sqsubseteq s \in \mathcal{R}} \neg V\left(\vec{r}, s, t_{1}, t_{2}\right) .
$$

Theorem 2. $\operatorname{CONS}(\mathcal{A L C D})$ is undecidable. 


\section{Reasoning on $\mathcal{E} \mathcal{L}$ with Dynamic Axioms}

Below we investigate the effects of adding DAs to $\mathcal{E} \mathcal{L}$ [Baader et al., 2005], ranging from tractability to undecidability.

\subsection{Positivity for $\mathcal{E} \mathcal{L}$ Leads to Tractability}

Since assertions and GCIs are atomic PDAs, we can restrict ourselves to checking the consistency of a DBox. Negation is not allowed in $\mathcal{E} \mathcal{L}$, hence any $\mathcal{E} \mathcal{L} \mathrm{KB}$ is trivially consistent. However, the consistency problem of $\mathcal{E} \mathcal{L}$ with positive DAs is no longer trivial. The following example demonstrates a way to obtain an inconsistent $\mathcal{E} \mathcal{L} \mathcal{D}^{+}$knowledge base: $\mathbb{U}_{\dagger}=$ $r(a, b) \sqcap(r(a, b) \circledast(T \sqsubseteq \top))$. Inconsistency happens because, if $\mathcal{I} \models \mathbb{U}_{\dagger}$, then there are $\mathcal{I}^{\prime}$ and $\mathcal{I}^{\prime \prime}$ such that $\mathcal{I}^{\prime}=\mathcal{I}+\mathcal{I}^{\prime \prime}$ and $\mathcal{I}^{\prime \prime} \models r(a, b)$. Moreover, as $\mathcal{I} \models \mathbb{U}_{\dagger}$, we have $\mathcal{I} \models r(a, b)$, which leads to a contradiction because $\mathcal{I}$ and $\mathcal{I}^{\prime \prime}$ have disjoint role interpretations. Below, we show that the inconsistency of PDAs can only be due to a generalisation of such a disjointness constraint. We provide an algorithm to decide $\operatorname{CONS}\left(\mathcal{E} \mathcal{L D}^{+}\right)$in polynomial time and we give technical developments for proving the correctness of our complexity characterisation. The proof for the adequateness of our algorithm reveals to be the difficult part. Let $\mathbb{U}$ be a PDA. In Figure 1, we define a simple proof system (parameterised by $\mathbb{U}$ ) that is essential to characterise the consistency status of $\mathbb{U}$. It takes care of the disjointness axioms in order to conclude whether $r(a, b)$ holds in the interpretation $\mathfrak{g}(\mathfrak{n})$. The calculus can derive either inconsistency $\perp$ or expressions of the form either $\mathfrak{n}: r(a, b)$ or $\mathfrak{n}: \neg r(a, b)$ where $\mathfrak{n} \in \operatorname{Int}_{\mathbb{U}}$ (such expressions are also called statements below). The expression $\mathfrak{n}: r(a, b)$ is intended to state that the interpretation associated with $\mathfrak{n}$ satisfies $r(a, b)$. We write $\mathbb{U} \vdash \mathfrak{n}: r(a, b)$ when $r(a, b)$ can be derived from the calculus with $\mathfrak{i}(\cdot)$ and Disj $\mathbb{U}_{\mathbb{U}}$ computed from $\mathbb{U}$ (similarly for $\mathfrak{n}: \neg r(a, b)$ and $\perp$ ).

Before presenting the first property of the proof system, given a positive dynamic axiom $\mathbb{U}$, we write $\mathcal{R} \mathcal{A}(\mathbb{U})$ to denote the set of role assertions $r(a, b)$ occurring in $\mathbb{U}$. A complete snapshot with respect to $\mathbb{U}$ is a map $\mathfrak{f}: \operatorname{lnt}_{\mathbb{U}} \times \mathcal{R} \mathcal{A}(\mathbb{U}) \rightarrow$ $\{0,1\}$ such that for all $r(a, b) \in \mathcal{R} \mathcal{A}(\mathbb{U})$,

- for all $\mathfrak{n} \in \operatorname{Int}_{\mathbb{U}}, \mathbb{U} \vdash \mathfrak{n}: r(a, b)$ implies $\mathfrak{f}(\mathfrak{n}, r(a, b))=1$, and $\mathbb{U} \vdash \mathfrak{n}: \neg r(a, b)$ implies $\mathfrak{f}(\mathfrak{n}, r(a, b))=0$,

- $\mathfrak{f}(\mathfrak{n}, r(a, b))=\mathfrak{f}\left(\mathfrak{n}_{1}, r(a, b)\right)+\mathfrak{f}\left(\mathfrak{n}_{2}, r(a, b)\right) \leq 1$, whenever $\mathfrak{n}=\mathfrak{n}_{1}+\mathfrak{n}_{2} \in$ Disj $_{\mathbb{U}}$.

Roughly speaking, a complete snapshot determines which pairs of domain elements are related by the interpretation of role names occurring in $\mathbb{U}$ (assuming the unique name assumption can be enforced, which will be the case). Obviously, $\mathfrak{f}(\mathfrak{n}, r(a, b))=1$ stands for $\mathfrak{g}(\mathfrak{n}) \models r(a, b)$ where $\mathfrak{g}$ is a complete witness. However, if $r, a$, and $b$ occur in $\mathbb{U}$ but not the role assertion $r(a, b)$, then there is no need to determine whether the interpretation associated with $\mathfrak{n}$ satisfies $r(a, b)$.

Lemma 5. Let $\mathbb{U}$ be a positive dynamic update s.t. $\mathbb{U} \forall \perp$. Then, there is a complete snapshot with respect to $\mathbb{U}$.

The constructive proof of Lemma 5 consists in building the complete snapshot by first using the statements $\alpha$ such that $\mathbb{U} \vdash \alpha$, then to set $\mathfrak{f}\left(\mathfrak{n}_{i}, r(a, b)\right)$ to 1 when $\mathfrak{n}=\mathfrak{n}_{1}+\mathfrak{n}_{2} \in$ $\operatorname{Disj}_{\mathbb{U}}, \mathfrak{f}(\mathfrak{n}, r(a, b))=1$, neither $\mathfrak{f}\left(\mathfrak{n}_{1}, r(a, b)\right)$ nor $\mathfrak{f}\left(\mathfrak{n}_{2}, r(a, b)\right)$ is defined, and $\mathfrak{n}_{i} \preceq \mathfrak{n}_{3-i}$. After removing these ambiguities,

$$
\begin{gathered}
\frac{\mathfrak{n}: r(a, b) \quad \mathfrak{n}: \neg r(a, b)}{\perp} \quad \frac{\mathfrak{f}_{\mathbb{U}}(\mathfrak{n})=r(a, b)}{\mathfrak{i}(\mathfrak{n}): r(a, b)} \\
\frac{\mathfrak{n}=\mathfrak{n}_{1}+\mathfrak{n}_{2} \in \operatorname{Disj}_{\mathbb{U}} \mathfrak{n}_{i}: r(a, b)}{\mathfrak{n}: r(a, b)} \\
\frac{\mathfrak{n}=\mathfrak{n}_{1}+\mathfrak{n}_{2} \in \operatorname{Disj}_{\mathbb{U}} \quad \mathfrak{n}_{i}: r(a, b)}{\mathfrak{n}_{3-i}: \neg r(a, b)} \\
\frac{\mathfrak{n}=\mathfrak{n}_{1}+\mathfrak{n}_{2} \in \operatorname{Disj}_{\mathbb{U}} \mathfrak{n}: r(a, b) \quad \mathfrak{n}_{i}: \neg r(a, b)}{\mathfrak{n}_{3-i}: r(a, b)} \\
\frac{\mathfrak{n}=\mathfrak{n}_{1}+\mathfrak{n}_{2} \in \operatorname{Disj}_{\mathbb{U}} \mathfrak{n}_{1}: \neg r(a, b) \quad \mathfrak{n}_{2}: \neg r(a, b)}{\mathfrak{n}: \neg r(a, b)} \\
\frac{\mathfrak{n}=\mathfrak{n}_{1}+\mathfrak{n}_{2} \in \operatorname{Disj}_{\mathbb{U}} \mathfrak{n}: \neg r(a, b)}{\mathfrak{n}_{i}: \neg r(a, b)}
\end{gathered}
$$

Figure 1: A simple proof system $(i \in\{1,2\})$.

$f$ is completed so that all the remaining values are 0 . One can check that $\mathfrak{f}$ is well-defined (in particular, it never happens that $\mathfrak{f}(\mathfrak{n}, r(a, b))=\mathbf{b}$ with $\mathbf{b} \in\{0,1\}$ and then set to $\mathfrak{f}(\mathfrak{n}, r(a, b))=(1-\mathbf{b}))$ and $\mathfrak{f}$ is a complete snapshot.

Lemma 6 (Correctness). Let $\mathbb{U}$ be a PDA s.t. $\mathbb{U} \vdash \mathfrak{n}: r(a, b)$ $($ resp. $\mathbb{U} \vdash \mathfrak{n}: \neg r(a, b)$ ). For all complete witnesses $\mathfrak{g}$ for $\mathbb{U}$, we have $\mathfrak{g}(\mathfrak{n}) \mid=r(a, b)($ resp. $\mathfrak{g}(\mathfrak{n}) \not \forall r(a, b))$.

As expected, the proof is by induction on the length of the derivation of $\mathbb{U} \vdash \alpha$ and by taking advantage of Lemma 1 . Consequently, if $\mathbb{U} \vdash \perp$, then any knowledge base $\mathcal{K}_{d a}=$ $(\mathcal{T}, \mathcal{A}, \mathcal{D})$ such that $\mathbb{U} \in \mathcal{D}$ is inconsistent. The proof system in Figure 1 is also complete, as stated below.

Lemma 7 (Characterisation). Let $\mathcal{K}_{d a}=(\mathcal{T}, \mathcal{A}, \mathcal{D})$ be a knowledge base with positive dynamic axioms. $\mathcal{K}_{d a}$ is consistent iff $\perp$ cannot be derived from $\mathbb{U}=\prod_{\alpha \in \mathcal{T} \cup \mathcal{A} \cup \mathcal{D}} \alpha$.

The assertions $C(a)$ play no role in the proof system and therefore such assertions have no influence on the derivability of $\perp$. This can be explained by the fact that any $\mathcal{E} \mathcal{L}$ knowledge base (without dynamic axioms) is consistent. Our characterisation is simple as it is sufficient to check the derivability of $\perp$. Though one direction of the proof is immediate from Lemma 6 , the other direction requires to build safely an interpretation satisfying $\mathcal{K}_{d a}$. To do so, we collect all the assertions and GCIs occurring in $\mathbb{U}$ and we know that their conjunction is consistent, say in some $\mathcal{I}$ satisfying the unique name assumption for all the individual names occurring in $\mathbb{U}$. From each domain element interpreting one such individual name, $\mathcal{I}$ is unravelled from it (using standard unravelling, see e.g. [Baader et al., 2017]) and copy a number of times polynomial in the size of $\mathbb{U}$. Unravellings and copies are performed to preserve the initial satisfaction on $\mathcal{I}$ whereas the different copies are designed so that we have enough edges between the domain elements to apply the dynamic connectives $*$ and - . The last step consists in deciding how to make true the assertions from $\mathcal{R A}(\mathbb{U})$. Lemma 5 is essential to make it in a consistent way. Indeed, the domain elements interpreting individual names occurring in $\mathbb{U}$ cannot be copied.

Theorem 3. $\operatorname{CONS}\left(\mathcal{E} \mathcal{L D}^{+}\right)$is in PTIME.

Given $\mathcal{K}_{d a}=(\mathcal{T}, \mathcal{A}, \mathcal{D})$, we build the equiconsistent PDA $\mathbb{U}=\prod_{\alpha \in \mathcal{T} \cup \mathcal{A} \cup \mathcal{D}} \alpha$. The computations of Tree $\mathbb{U}$, Int $\mathbb{U}$ 
and Disj $\underset{\Psi}{ }$ can be done in PTIME. Determining which statements are derivable from $\mathbb{U}$ can be also done in PTIME, by using a saturation algorithm and observing that the number of potential derivable statements is polynomial too.

\subsection{Why Being Positive is Always Better}

In this section, we show that $\operatorname{CONS}(\mathcal{E} \mathcal{L} \mathcal{D})$ is undecidable, by refining the arguments for the undecidability of $\operatorname{CONS}(\mathcal{A L C D})$. The main difficulty is to encode concept negation with the help of dynamic axioms (not necessarily positive) but when the concepts are restricted to those from $\mathcal{E} \mathcal{L}$. Technical developments are mainly based on those from Section 4.2. Below, we pinpoint the main differences.

We use an auxiliary vocabulary including the concept name $E m p$ and the role names $t, t_{1}, t_{2}, t_{3}$ that are reserved to define auxiliary concepts and dynamic axioms. W.l.o.g., we assume that these names do not occur in our instances of $\mathcal{A L C}$ augmented with CRIAs. Roughly speaking, in the translation, we force $E m p$ to behave as $\perp$ from $\mathcal{A L C}$. Recall that $\perp$ is not an $\mathcal{E} \mathcal{L}$ concept, and it is heavily used in the undecidability proof from Section 4.2. To provide the right semantics of $E m p$, we introduce the dynamic axiom [Emp $\left.\equiv_{t} \perp\right]$ defined as $\left[E m p \equiv_{t} \perp\right] \stackrel{\text { def }}{=} \neg(\top \circledast(\top \sqsubseteq \exists t . E m p))$.

Lemma 8. For every $\mathcal{I}, E m p^{\mathcal{I}}=\emptyset$ iff $\mathcal{I} \models\left[E m p \equiv_{t} \perp\right]$.

So, for any arbitrary $\mathcal{E} \mathcal{L}$ concept $C$, assuming that $\mathcal{I} \models$ $\left[E m p \equiv_{t} \perp\right]$, we have $\mathcal{I}=C \sqsubseteq E m p$ iff $C^{\mathcal{I}}=\emptyset$. Moreover, $E m p$ allows us to redefine in $\mathcal{E} \mathcal{L D}\left\langle C \neq_{t} \perp\right\rangle$ introduced for $\mathcal{A L C D}$ : it is sufficient to substitute $\perp$ with Emp. Indeed, assuming that the role names occurring in $C$ are from $R$, and $t \notin R$, then the $\mathcal{E} \mathcal{L} \mathcal{D}$ PDA below

$$
\left\langle C \not \equiv_{t} \perp\right\rangle \stackrel{\text { def }}{=}\left(\prod_{r \in R}(\exists r . \top \sqsubseteq E m p)\right) \circledast(\top \sqsubseteq \exists t . C)
$$

satisfies the following lemma (analogous to Lemma 3 ).

Lemma 9. Let $\mathcal{I}$ be an interpretation such that $E m p^{\mathcal{I}}=\emptyset$ and $t^{\mathcal{I}}=\emptyset$. We have $\mathcal{I}=\left\langle C \neq_{t} \perp\right\rangle$ iff $C^{\mathcal{I}} \neq \emptyset$.

The dynamic axiom $V\left(\vec{r}, s, t_{1}, t_{2}\right)$ introduced in Section 4.2 to express a CRIA $\vec{r} \sqsubseteq s$ can be translated into $\mathcal{E} \mathcal{L} \mathcal{D}$. Again, every occurrence of $\perp$ is substituted with Emp. However, this is not enough as we need to deal with the negated concept $\neg \exists s . \exists t_{1} . \top$ from $V\left(\vec{r}, s, t_{1}, t_{2}\right)$ in Section 4.2. To do so, we introduce an auxiliary role name $t_{3}$ and simply state that the first projections of $\left(s \circ t_{1}\right)^{\mathcal{I}}$ and $r_{3}^{\mathcal{I}}$ are disjoint, but this is not the case for $\left(r_{1} \circ \cdots \circ r_{n} \circ t_{1}\right)^{\mathcal{I}}$ and $r_{3}^{\mathcal{I}}$. The resulting formula $V\left(\vec{r}, s, t_{1}, t_{2}, t_{3}\right)$ is defined as follows:

$$
\begin{array}{r}
\left(\prod \exists r . \top \sqsubseteq E m p\right) \circledast\left(\left(\exists t_{2} \cdot \top \sqcap \exists s . \exists t_{1} \cdot \top\right) \sqsubseteq E m p\right) \sqcap \\
\left.r \in\left\{r_{1}, \ldots, r_{n}, s, t_{3}\right\} \quad\left\langle\exists r_{1} \ldots . \exists r_{n} \cdot \exists t_{1} \cdot \top \sqcap \exists t_{2} \cdot \top \not \equiv t_{3} \perp\right\rangle\right) .
\end{array}
$$

Lemma 10 (closely related to Lemma 4) states that this formula behaves as its homonymous formula in $\mathcal{A L C D}$.

Lemma 10. Let $\mathcal{I}$ be an interpretation s.t. $E m p^{\mathcal{I}}=\emptyset$ and for every $i \in\{1,2,3\} t_{i}^{\mathcal{I}}=\emptyset$. Let $\vec{r} \sqsubseteq s$ be a CRIA, where $\vec{r}=r_{1} \circ \cdots \circ r_{n}$. Then, $\mathcal{I} \models \neg V\left(\vec{r}, s, t_{1}, t_{2}, t_{3}\right)$ iff $\mathcal{I} \models \vec{r} \sqsubseteq s$.

Now, let $C$ be an $\mathcal{A L C}$ concept, $\mathcal{R}$ be an RBox and $a$ be an arbitrary individual name. Let us assume for the moment that the assertion $C(a)$ can be translated into an equivalent DA $\tau(C(a))$ of $\mathcal{E} \mathcal{L} \mathcal{D}$ (shown below). Then, by relying on the formulae defined previously, we conclude that $C$ is satisfiable with respect to $\mathcal{R}$ iff the DA in $\mathcal{E} \mathcal{L} \mathcal{D}$ below is consistent:

$$
\begin{aligned}
\tau(C(a)) \sqcap\left[E m p \equiv_{t_{1}} \perp\right] & \sqcap \prod_{i \in[1,3]}\left(\exists t_{i} \cdot \top \sqsubseteq E m p\right) \\
& \sqcap \prod_{\vec{r} \sqsubseteq s \in \mathcal{R}} \neg V\left(\vec{r}, s, t_{1}, t_{2}, t_{3}\right) .
\end{aligned}
$$

It remains to explain how to replace $C(a)$ by a DA $\tau(C(a))$ in $\mathcal{E} \mathcal{L} \mathcal{D}$, while preserving the equivalence. We use the renaming technique [Scott, 1962] and rely on the presence of GCIs in DAs. Let $D_{1}, \ldots, D_{\alpha}$ be the subconcepts of $C$, say $D_{1}=C$. We use new concept names $B_{1}, \ldots, B_{\alpha}$ not appearing in $C$. The idea is to enforce a dynamic axiom $U_{i}$, for all $i \in[1, \alpha]$, that glues the new concept names in a way that $B_{i}$ is shown equivalent to $D_{i}$. Formally, these DAs are computed as follows $\left(C_{1} \equiv C_{1}^{\prime}\right.$ denotes $\left.\left(C_{1} \sqsubseteq C_{1}^{\prime}\right) \sqcap\left(C_{1}^{\prime} \sqsubseteq C_{1}\right)\right)$.

- If $D_{i}$ is a concept name or $\top$, then $U_{i} \stackrel{\text { def }}{=}\left(B_{i} \equiv D_{i}\right)$,

- If $D_{i}=D_{j} \sqcap D_{k}$ then $U_{i} \stackrel{\text { def }}{=}\left(B_{i} \equiv\left(B_{j} \sqcap B_{k}\right)\right)$,

- If $D_{i}=\exists r_{k} \cdot D_{j}$ then $U_{i} \stackrel{\text { def }}{=}\left(B_{i} \equiv \exists r_{k} \cdot B_{j}\right)$,

- $U_{i} \stackrel{\text { def }}{=}\left[\top \sqsubseteq B_{i} \sqcup B_{j}\right]_{t_{1}} \sqcap \neg\left(\top \circledast\left(\top \sqsubseteq \exists t_{1} \cdot\left(B_{i} \sqcap B_{j}\right)\right)\right)$ whenever $D_{i}=\neg D_{j}$, where $\left[\top \sqsubseteq B_{i} \sqcup B_{j}\right]_{t_{1}}$ denotes

$$
\begin{aligned}
& \neg\left(\exists t_{1} \cdot \top \sqsubseteq E m p-\left(\left\langle\exists t_{2} \cdot \top \not z_{t_{1}} \perp\right\rangle \sqcap\right.\right. \\
& \left.\left.\quad\left(B_{i} \sqcap \exists t_{2} \cdot \top \sqsubseteq E m p\right) \sqcap\left(B_{j} \sqcap \exists t_{2} \cdot \top \sqsubseteq E m p\right)\right)\right) .
\end{aligned}
$$

We define $\tau(C(a))$ as $B_{1}(a) \sqcap\left(\prod_{i \in[1, \alpha]} U_{i}\right)$, completing the definition of the equiconsistent $*$-free dynamic axiom. We are now in position to state a striking undecidability result.

Theorem 4. $\operatorname{CONS}(\mathcal{E} \mathcal{L} \mathcal{D})$ is undecidable (even without $*$ ).

\section{Concluding Remarks}

We introduced a framework for enriching knowledge bases with a dynamic box (DBox) that specifies how the satisfaction of GCIs and assertions evolves when the interpretation is modified. Its dynamic connectives $*$ and $\circledast$ are imported from separation logics. Such an incursion of separation logics in DLs is completely new. The framework can be developed in many directions but we focused on a few landmark problems. Whereas we have shown that $\operatorname{CONS}\left(\mathcal{A L C D}{ }^{+}\right)$is ExPTIME-complete and $\operatorname{CONS}\left(\mathcal{E} \mathcal{L D}^{+}\right)$is in PTIME, the extensions with dynamic axioms admitting negation at the top level lead to undecidable problems. The PTIME upper bound for $\operatorname{CONS}\left(\mathcal{E} \mathcal{L} \mathcal{D}^{+}\right)$is obtained using a simple algorithm thanks to a characterisation whose proof requires a fine-tuned model-theoretical construction. Besides, we showed that the satisfiability problem for $\mathcal{A L C}$ with respect to complex role inclusion axioms can be reduced to $\operatorname{CONS}(\mathcal{A L C D})$, leading to undecidability. More surprisingly, $\operatorname{CONS}(\mathcal{E} \mathcal{L} \mathcal{D})$ is undecidable too (which amounts to encode concept negation).

Though updates for ABoxes have been already investigated, see e.g. [Liu et al., 2006; De Giacomo et al., 2006], our framework is original as it concerns updates in interpretations. In the paper, we only considered (de)composition of roles and the only reasoning task was about knowledge base consistency. Other variants are possible (domain (de)composition, subsumption problems, etc.) in particular related to alternative interpretations for the composition operator.

\section{Acknowledgements}

B. Bednarczyk is supported by ERC Consolidator Grant 771779 (DeciGUT). S. Demri and A. Mansutti are supported by the Centre National de la Recherche Scientifique (CNRS). 


\section{References}

[Baader et al., 2005] F. Baader, S. Brandt, and C. Lutz. Pushing the EL envelope. In IJCAI'05, pages 364-369. Professional Book Center, 2005.

[Baader et al., 2017] F. Baader, I. Horrocks, C. Lutz, and U. Sattler. An Introduction to Description Logic. Cambridge University Press, 2017.

[Baldoni et al., 1998] M. Baldoni, L. Giordano, and A. Martelli. A tableau calculus for multimodal logics and some (un)decidability results. In TABLEAUX'98, volume 1397 of $L N A I$, pages 44-59. Springer, 1998.

[Bozzelli et al., 2013] L. Bozzelli, H. van Ditmarsch, and S. Pinchinat. The complexity of one-agent refinement modal logic. In IJCAI'13, pages 2977-2981, 2013.

[Calvanese et al., 2009] D. Calvanese, T. Eiter, and M. Ortiz. Regular path queries in expressive description logics with nominals. In IJCAI'09, pages 714-720, 2009.

[Calvanese et al., 2014] D. Calvanese, T. Kotek, M. Simkus, H. Veith, and F. Zuleger. Shape and content - A databasetheoretic perspective on the analysis of data structures. In IFM'14, volume 8739 of $L N C S$, pages 3-17. Springer, 2014.

[De Giacomo et al., 2006] G. De Giacomo, M. Lenzerini, A. Poggi, and R. Rosati. On the update of description logic ontologies at the instance level. In AAAI'06, pages 12711276. AAAI Press, 2006.

[De Giacomo et al., 2017] G. De Giacomo, D. Lembo, X. Oriol, D. Fabio Savo, and E. Teniente. Practical Update Management in Ontology-Based Data Access. In ISWC'17, volume 10587 of LNCS, pages 225-242. Springer, 2017.

[Demri, 2001] S. Demri. The complexity of regularity in grammar logics and related modal logics. JLC, 11(6):933960, 2001.

[Distefano et al., 2019] D. Distefano, M. Fähndrich, F. Logozzo, and P.W. O'Hearn. Scaling static analyses at Facebook. Commun. ACM, 62(8):62-70, 2019.

[Drescher et al., 2009] C. Drescher, H. Liu, F. Baader, S. Guhlemann, U. Petersohn, P. Steinke, and M. Thielscher. Putting ABox updates into action. In FroCoS'09, volume 5749 of $L N C S$, pages 214-229. Springer, 2009.

[Georgieva and Maier, 2005] L. Georgieva and P. Maier. Description logics for shape analysis. In SEFM'05, pages 321-331. IEEE Computer Society, 2005.

[Horrocks and Sattler, 2004] I. Horrocks and U. Sattler. Decidability of SHIQ with complex role inclusion axioms. AI, 160(1-2):79-104, 2004.

[Liu et al., 2006] H. Liu, C. Lutz, M. Milicić, and F. Wolter. Updating Description Logic ABoxes. In KR'06, pages 4656. AAAI Press, 2006.

[Plaza, 1989] J. Plaza. Logics of public communication. In ISMIS'89, Charlotte, North Carolina, USA, 1989.
[Reynolds, 2002] J.C. Reynolds. Separation logic: a logic for shared mutable data structures. In $\mathrm{LiCS}^{\prime} 02$, pages 55-74. IEEE, 2002.

[Scott, 1962] D. Scott. A decision method for validity of sentences in two variables. The Journal of Symbolic Logic, 27:377, 1962.

[Vafeiadis and Parkinson, 2007] V. Vafeiadis and M. Parkinson. A Marriage of Rely/Guarantee and Separation Logic. In CONCUR'07, volume 4703 of LNCS, pages 256-271. Springer, 2007.

[van Benthem, 2005] J. van Benthem. An Essay on Sabotage and Obstruction. In Mechanizing Mathematical Reasoning, Essays in Honor of Jörg Siekmann on the Occasion of his 69th Birthday, pages 268-276. Springer Verlag, 2005. 